\title{
Revisiting monotop production at the LHC
}

\author{
Idir Boucheneb, ${ }^{a}$ Giacomo Cacciapaglia, ${ }^{a, b}$ Aldo Deandrea ${ }^{a, b, 1}$ and Benjamin Fuks ${ }^{c, d}$ \\ ${ }^{a}$ Université de Lyon, Université Lyon 1, \\ F-69622 Villeurbanne, France \\ ${ }^{b}$ Institut de Physique Nucléaire de Lyon, CNRS/IN2P3, UMR5822, \\ F-69622 Villeurbanne Cedex, France \\ ${ }^{c} \mathrm{CERN}, \mathrm{PH}-\mathrm{TH}$, \\ CH-1211 Geneva 23, Switzerland \\ ${ }^{d}$ Institut Pluridisciplinaire Hubert Curien/Département Recherches Subatomiques, \\ Université de Strasbourg/CNRS-IN2P3, 23 rue du Loess, F-67037 Strasbourg, France \\ E-mail: idir.boucheneb@gmail.com, g.cacciapaglia@ipnl.in2p3.fr, \\ deandrea@ipnl.in2p3.fr, benjamin.fuks@cern.ch
}

AbStRACT: Scenarios of new physics where a single top quark can be produced in association with large missing energy (monotop) have been recently studied both from the theoretical point of view and by experimental collaborations. We revisit the originally proposed monotop setup by embedding the effective couplings of the top quark in an $\mathrm{SU}(2)_{L}$ invariant formalism. We show that minimality selects one model for each of the possible production mechanisms: a scalar field coupling to a right-handed top quark and an invisible fermion when the monotop system is resonantly produced, and a vector field mediating the interactions of a dark sector to right-handed quarks for the non-resonant production mode. We study in detail constraints on the second class of scenarios, originating from contributions to standard single top processes when the mediator is lighter than the top quark and from the dark matter relic abundance when the mediator is heavier than the top quark.

Keywords: Phenomenological Models

ArXiv EPrint: 1407.7529

\footnotetext{
${ }^{1}$ Also at: Institut Universitaire de France, 103 boulevard Saint-Michel, 75005 Paris, France.
} 


\section{Contents}

1 Introduction 1

2 Gauge-invariant effective Lagrangians for monotop production 3

2.1 Resonant monotop production 3

2.2 Non-resonant monotop production 6

3 Monotop phenomenology specific to non-resonant models 10

$\begin{array}{ll}3.1 \text { Mediators heavier than the top quark } & 10\end{array}$

$\begin{array}{lll}3.1 .1 & \text { Tree-level decays of the mediator } & 10\end{array}$

$\begin{array}{lll}\text { 3.1.2 Dark matter constraints } & 11\end{array}$

$\begin{array}{ll}3.2 & \text { Mediators lighter than the top quark } \\ 3.2 .14\end{array}$

$\begin{array}{lll}3.2 .1 & \text { Loop-induced and multibody decays of the mediator } & 15\end{array}$

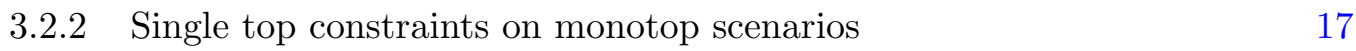

$\begin{array}{lll}3.2 .3 & \text { Dark matter constraints } & 19\end{array}$

4 Conclusions $\quad 20$

\section{Introduction}

The first phase of the LHC experiments has given two important messages: a scalar resonance closely resembling the Standard Model Higgs boson has been discovered, and new physics beyond the Standard Model has not been found. The latter result would imply that new states or effects beyond the Standard Model predictions may be much more difficult to spot at the LHC than we previously thought. In fact, very strong bounds have been posed on easy-catch models, like the constrained version of the Minimal Supersymmetric Standard Model [1, 2].

Many theorists have therefore recently turned their attention on a more signature-based strategy, focusing on unusual final states which are difficult to detect or have not been considered yet by experimental collaborations. One such final state that has been gaining popularity among phenomenologists [3-17] and experimentalists [18-21] is the monotop signature: a single top quark produced in association with a large amount of missing energy. Although the production of this final state is very suppressed in the Standard Model, it is however not easy to obtain this kind of events in realistic and complete models of new physics. Two main production mechanisms can lead to a monotop state [8, 15], arising either from the resonant production of a coloured bosonic state which further decays into a top quark plus an invisible neutral fermion; or via the production of a single top quark in association with an invisible boson that has flavour-changing couplings to top and light quarks. Examples of the first class of models include $R$-parity violating supersymmetry, 
where the produced resonance is a top squark decaying into a top plus a long-lived neutralino $[3,5,6,12]$. The second class of models has been described in scenarios of dark matter from a hidden sector that couples to the Standard Model via flavour-violating couplings of a bosonic mediator [7, 9, 14, 22]. In general, monotop signatures can be however generated by other processes involving, for instance, the $t$-channel exchange of a new particle, different spin assigments for the new states or higher-spin tensors. Motivated by the setups currently under study experimentally, this work is limited to the case of a spin-0 or spin-1 state that can be either exchanged in the $s$-channel (named "resonant" in the following) or produced in association with a top quark via flavour-changing interactions (named "non-resonant").

All such models can be described in terms of a simple Lagrangian [8], which contains all the possible couplings giving rise to a monotop signal. A very general analysis of this framework can be found in ref. [15], the limiting case of higher-dimensional operators has been discussed in ref. [10], while monotop production via flavour-changing interactions of quarks with an invisible $Z$-boson has been detailed in ref. [4]. Although this simple description has the advantage of being complete, it has the drawback of containing too many free parameters to be efficiently scanned by an experimental search. Furthermore, the included couplings do not respect the symmetries of the Standard Model, as they are intended to describe the model dynamics after the breaking of the electroweak symmetry. In this way, this approach ignores other interactions needed to restore gauge invariance which can give rise to new physics signals in different search channels, the latter possibly implying stronger constraints on the parameters of the model than the monotop search itself. In this work, we revisit the parametrisation originally proposed in ref. [8] by paying particular attention to the embedding of the Lagrangian description into $\mathrm{SU}(2)_{L} \times \mathrm{U}(1)_{Y}$ invariant operators. We therefore present a set of minimal effective Lagrangians, extending the Standard Model with gauge-invariant operators. Our approach allows us to restrict the number of "interesting" scenarios, i.e., the cases where the monotop signal is genuinely the main signal of new physics to be expected at the LHC. Equivalently, this reduces the number of free parameters to a manageable number. Finally, we discuss in detail how the effective model could be completed in order to guarantee that the missing energy particle produced in association with the top quark is indeed either long-lived or decaying into invisible states.

The rest of this work is organized as follows. In section 2, we present how to construct gauge-invariant effective models for the monotop signal. We consider separately the resonant case, where the mediator is a scalar or vector boson, and the non resonant case where the top is produced in association with a scalar or vector via flavour-changing couplings. We also discuss how the effective Lagrangians can be matched to the simple monotop descriptions of ref. [8]. We then focus on non-resonant scenarios which turn out to be less "standard" and investigate, in section 3, the conditions under which the invisible state is effectively invisible, and other experimental observations, which can further constrain the model. Our conclusions are presented in section 4. 


\section{Gauge-invariant effective Lagrangians for monotop production}

\subsection{Resonant monotop production}

In the first class of scenarios yielding the production of a monotop system at colliders that we consider, the produced top quark recoils against an invisible fermionic state $\chi$. Being singly produced, the $\chi$ particle cannot be stable, thus it is either long-lived or it decays into a pair of stable particles. In each case, it has to be electrically-neutral and a coloursinglet. In resonant monotop production, both final-state particles arise from the decay of a heavy scalar $\varphi$ or vector $X$ field, lying in the fundamental representation of $\mathrm{SU}(3)_{c}$, that is produced in the $s$-channel from the fusion of two down-type (anti-)quarks. The parton-level process that we want to focus on is therefore

$$
d d \rightarrow \varphi, X_{\mu} \rightarrow \bar{t} \chi .
$$

In order to understand how the scalar or vector mediators transform under the full Stanard Model symmetries, it is crucial to define the chirality of the quarks it couples too. In the following we will analyse separately the scalar and vector case in detail.

Spin-0 mediator. The initial state consists of a pair of down-type quarks which form a spin-0 state. The related Lorentz scalar fermionic bilinear reads $\bar{\psi} \psi$, which can be written as $\bar{\psi}_{L} \psi_{R}+\bar{\psi}_{R} \psi_{L}$. This implies that the two quarks have opposite chiralities. Recalling that the charge conjugate of the right-handed quark $d_{R}^{C}$ is left-handed while the one of the left-handed quark $d_{L}^{C}$ is right-handed, we can define two independent initial states, one with explicit right-handed and one with left-handed chirality indices. According to the Standard Model gauge symmetries $\mathrm{SU}(3)_{c}, \mathrm{SU}(2)_{L}$ and $\mathrm{U}(1)_{Y}$, the two states transform as:

$$
\bar{d}_{R}^{C i} d_{R}^{j}=(\underset{\sim}{\overline{\mathbf{3}}}, \underset{\sim}{\mathbf{1}},-2 / 3) ; \quad \bar{d}_{L}^{C}{ }^{i} d_{L}^{j}=(\underset{\sim}{\overline{\mathbf{3}}}, \underset{\sim}{\mathbf{3}}, 1 / 3) .
$$

In our notation, an undertilde indicates a representation under a non-Abelian gauge symmetry, and the indices $i, j$ refer to flavour. Since the diquark states are made of identical quarks and fermions are anticommuting quantities, the corresponding wavefunctions need to be antisymmetric under the exchange of the quark fields. The exchange of the flavour indices is therefore forced to be antisymmetric too, since the one of the spin and colour indices (which we do not explicit for simplicity) are antisymmetric and the one of the triplet (adjoint) representation of $\mathrm{SU}(2)_{L}$ is symmetric (for the left-handed quark setup). From the representations shown above, the right and left-handed quarks cannot couple to the same scalar and two different objects must thus be introduced,

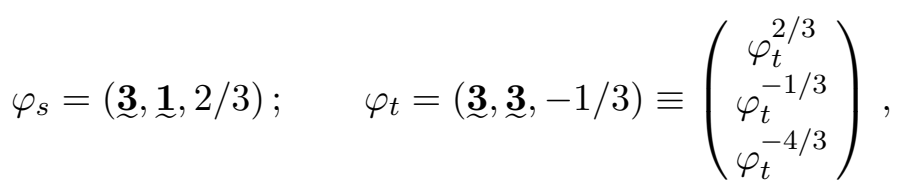

where the subscript ${ }_{s}$ and ${ }_{t}$ refer to singlet and triplet of $\mathrm{SU}(2)_{L}$. The operators containing the interactions needed for monotop production can be written as

$$
\lambda_{s} \varphi_{s} \bar{d}_{R}^{C} d_{R}+\lambda_{t} \varphi_{t} \bar{q}_{L}^{C} q_{L}+\text { h.c. },
$$


where $q_{L}$ is the left-handed doublet, and $\lambda_{s, t}$ are antisymmetric matrices in flavour space. In the second term, it is the component of the triplet with electric charge $\pm 2 / 3$ that couples eventually to the top.

We can now repeat the same analysis for the final state. We first assume, for minimality, that the invisible fermion $\chi$ is a singlet under the Standard Model symmetries. In this case, the final state system representation under the Standard Model gauge group can be

$$
\chi_{R} t_{R}=(\underset{\sim}{\mathbf{3}} \underset{\sim}{\mathbf{1}}, 2 / 3) ; \quad \chi_{L} t_{L}=(\underset{\sim}{\mathbf{3}}, \underset{\sim}{\mathbf{2}}, 1 / 6) .
$$

In order to allow for a $\chi$-coupling to a left-handed top quark, we therefore need to introduce an extra scalar field $\varphi_{d}$, compared to eq. (2.3), which transforms as a doublet of $\mathrm{SU}(2)_{L}$. The operators relevant for monotop production can then be written as

$$
y_{s} \varphi_{s}^{\dagger} \bar{\chi} t_{R}+y_{d} \varphi_{d}^{\dagger} \bar{\chi} q_{L}+\text { h.c. }
$$

The initial and final state can consequently only be connected via an $\mathrm{SU}(2)_{L}$-singlet field $\varphi_{s}$ that couples to right-handed quarks. This scenario being minimal, it will therefore be considered in the rest of this work. For completeness, let us mention that non-minimal models with several additional scalars could also be constructed. In these setups, the new scalar fields are allowed to mix after electroweak symmetry breaking through couplings to the Brout-Englert-Higgs field $\phi_{H}=(\underset{\sim}{\mathbf{1}}, \mathbf{2}, 1 / 2)$,

$$
\mu_{t} \phi_{H}^{\dagger} \varphi_{t}^{\dagger} \varphi_{d}+\mu_{d} \varphi_{s} \varphi_{d}^{\dagger} \phi_{H}^{\dagger}+\text { h.c. }
$$

The resulting mass splitting is nevertheless constrained to be small by the perturbativity of the couplings [23] and corrections to the $S$ and $T$ parameters [24, 25].

We have also imposed that the $\chi$-field has the same quantum numbers as a right-handed neutrino, so that it could potentially mix with neutrinos. This mixing is however strongly constrained by proton-decay processes like $p \rightarrow \pi^{+} / K^{+} \nu$. In this case, the contribution of the box-diagram-induced subprocess $d u \rightarrow \bar{d} / \bar{s} \nu$ (through a $\varphi_{s}$ and $W^{+}$exchange) has indeed to be maintained small. There is however a way to evade the bound by preventing $\chi$ from mixing with neutrinos (by assigning it, e.g., a baryon number), unless $\chi$ is lighter than the proton.

We have considered so far models where the invisible fermion $\chi$ is a Standard Model gauge singlet. Another option would be to assume that $\chi$ is the neutral component of a non-trivial $\mathrm{SU}(2)_{L}$ multiplet. For instance, one could choose (with $\sigma_{2}$ being the second Pauli matrix)

$$
\begin{aligned}
\chi_{d}=(\underset{\sim}{\mathbf{1}} \underset{\sim}{\mathbf{2}}, 1 / 2) \equiv\left(\begin{array}{c}
\chi^{+} \\
\chi^{0}
\end{array}\right) \quad \Rightarrow \quad \chi_{L} t_{L}=(\underset{\sim}{\mathbf{3}}, \underset{\sim}{\mathbf{1}}, 2 / 3), \\
\chi_{d}^{\prime}=i \sigma_{2} \chi_{d}^{*}=(\underset{\sim}{\mathbf{1}} \underset{\sim}{\mathbf{2}},-1 / 2) \equiv\left(\begin{array}{c}
\chi^{0} \\
\chi^{-}
\end{array}\right) \quad \Rightarrow \quad \chi_{L} t_{L}=(\underset{\sim}{\mathbf{3}} \underset{\sim}{\mathbf{3}},-1 / 3), \\
\chi_{t}=(\underset{\sim}{\mathbf{1}}, \underset{\sim}{\mathbf{3}},-1) \equiv\left(\begin{array}{c}
\chi_{0} \\
\chi^{-} \\
\chi^{--}
\end{array}\right) \quad \Rightarrow \quad \chi_{R} t_{R}=(\underset{\sim}{\mathbf{3}}, \underset{\sim}{\mathbf{3}},-1 / 3) .
\end{aligned}
$$


For the sake of the example, we focus on the first option where monotop systems can be produced via the production and the decay of a $\varphi_{s}$ resonance. The presence of charged degrees of freedom in the $\chi$ multiplet allows one to constrain this scenario by other sources like, for instance, single bottom production $\bar{d}_{R} \bar{d}_{R} \rightarrow \varphi_{s} \rightarrow b \chi^{+}$. The mass splitting between the neutral and charged component of the $\chi_{d}$ doublet being generated by electroweak loopdiagrams (unless they mix to other fermions), the decay of the charged $\chi^{+}$field is driven by the two competing channels $\chi^{+} \rightarrow \bar{b}_{L} \bar{d}_{R}^{i} \bar{d}_{R}^{j}$ (mediated by $\varphi_{s}$ ) and $\chi^{+} \rightarrow \chi_{0} W^{*}$ (with a very off-shell $W$-boson). As a result, the $\chi^{+}$particle is in general long-lived, which is heavily constrained by current LHC searches $[26,27]$. Furthermore, $\chi_{d}$ has the same quantum numbers as the lepton doublets of the Standard Model so that these fields can mix, which induces the proton decay modes $p \rightarrow \pi^{+} / K^{+} \nu$ and $p \rightarrow \pi^{0} / K^{0} e^{+}$similarly as described above. Consequently, it turns out that scenarios where the invisible $\chi$ fermion is one of the components of a larger $\mathrm{SU}(2)_{L}$ multiplet are unlikely to be realized. Although we will ignore those non-minimal scenarios, their complete analysis is however in order, which goes beyond the scope of this work focusing on monotop production only.

Spin-1 mediator. We now turn to cases where monotop systems are produced from the decay of a spin-1 resonance $X$. The related Lorentz vector fermionic bilinear is given by $\bar{\psi} \gamma_{\mu} \psi$, which can be written as $\bar{\psi}_{L} \gamma_{\mu} \psi_{L}+\bar{\psi}_{R} \gamma_{\mu} \psi_{R}$. This implies that the two quarks have the same chirality. In order to build a scalar invariant, vector fields have to couple to these spinors of the same chiralities. Using the same properties of the charge conjugation used in the notations for the scalar case, the possible couplings of the $X$-field to down-type quarks are then of the form

$$
\lambda_{V}^{1} X^{\mu} \bar{d}_{L}^{C} \gamma_{\mu} d_{R}+\text { h.c. }
$$

where we denote the coupling strength by $\lambda_{V}$. In order for such couplings to be $\mathrm{SU}(2)_{L^{-}}$ invariant, the $X$-boson must belong to a weak doublet with hypercharge $1 / 6$,

$$
X_{\mu}=(\underset{\sim}{\mathbf{3}}, \underset{\sim}{\mathbf{2}}, 1 / 6) \equiv\left(\begin{array}{c}
X_{\mu}^{2 / 3} \\
X_{\mu}^{-1 / 3}
\end{array}\right) .
$$

Turning to the final state, we begin with the fact that the $X$-field defined above has the quantum numbers of a left-handed quark doublet. It can consequently couple to a left-handed top quark and a singlet field $\chi$,

$$
\lambda_{V}^{2} X_{\mu} \bar{q}_{L} \gamma^{\mu} \chi+\text { h.c. }
$$

Enforcing weak isospin gauge invariance implies thus, in addition to the interaction relevant for the production of a monotop state, the presence of the interaction of a left-handed bottom quark to the second component of the $X$-doublet. This however induces the fast decay of the neutral $\chi$ fermion via an off-shell $X$-state,

$$
\chi \rightarrow b_{L}\left(X_{\mu}^{-1 / 3}\right)^{*} \rightarrow b_{L} u_{L} d_{R} \quad\left(\text { or } \quad \bar{b}_{L} \bar{u}_{L} \bar{d}_{R} \text { if } \chi \text { is a Majorana fermion }\right)
$$


so that this model does not predict any monotop signal. We therefore move on with a second option for linking the $\chi$ - $t$ monotop system to the $X$-boson by considering righthanded quarks. A coupling to right-handed quarks can be obtained if the fermion $\chi$ belongs to an $\mathrm{SU}(2)_{L}$ doublet with hypercharge $1 / 2, \chi_{d}=(\underset{\sim}{\mathbf{1}}, \underset{\sim}{\mathbf{2}}, 1 / 2)$,

$$
\lambda_{V}^{3} X_{\mu} \bar{t}_{R} \gamma^{\mu} \chi_{d}+\text { h.c. }
$$

This model however contains a charged fermion that can be produced in association with a top via the vector of charge $-1 / 3$ and is therefore likely to be constrained by channels different from the monotop one.

More complicated non-minimal possibilities could also be considered but will be ignored from this work for the same reason: they are strongly constrained by other processes and their analysis must account for these other channels, in addition to the monotop signature.

Summary for the resonant channel. From the analysis of gauge invariant effective Lagrangians performed in this subsection, we have shown that the chirality of both the initial down-type quarks and the final-state top quark are correlated with the quantum numbers allowed for the bosonic mediator and the invisible fermion. In this work, we focus on the minimal model in terms of field content and interactions. In this case, the setup that predicts monotop production at the LHC as its main signature (and that is thus not constrained by any other observable) contains a scalar mediator and a new fermion that are both gauge-singlet and couple to right-handed quarks. The effective Lagrangian reads

$$
\mathcal{L}_{\text {eff. }}=\mathcal{L}_{\text {kin }}\left(\varphi_{s}, \chi\right)+\lambda_{s}^{i j} \varphi_{s} \bar{d}_{R, i}^{C} d_{R, j}+y_{s} \varphi_{s}^{\dagger} \bar{\chi} t_{R}+\text { h.c. },
$$

where $\mathcal{L}_{\text {kin }}$ contains gauge interaction, kinetic and mass terms for the new fields, and the other terms focus on their interactions with the Standard Model quarks. This can be compared to the notation of ref. [8] where the Lagrangian describing the same scenario is written as

$$
\mathcal{L}_{\text {res }}=\mathcal{L}_{\text {kin }}\left(\varphi_{s}, \chi\right)+\left(\varphi \bar{d}_{i}^{C}\left[\left(a_{S R}^{q}\right)^{i j}+\left(b_{S R}^{q}\right)^{i j} \gamma^{5}\right] d_{j}+\varphi \bar{t}\left[a_{S R}^{1 / 2}+b_{S R}^{1 / 2} \gamma^{5}\right] \chi+\text { h.c. }\right),
$$

flavour indices being noted by $i, j$ and colour indices being omitted for clarity. The two Lagrangians are related by

$$
a_{S R}^{q}=b_{S R}^{q}=\lambda_{s} / 2, \quad a_{S R}^{1 / 2}=b_{S R}^{1 / 2}=y_{s}^{*} / 2 .
$$

As already mentioned, the couplings of the scalar to the down quarks are antisymmetric under the exchange of the flavour indices. Consequently, parton density effects enhance the production mode $d s \rightarrow \varphi^{*}$ at hadron colliders (with the relevant coupling strengths being non-vanishing), as already pointed out in previous works $[8,12,15]$.

\subsection{Non-resonant monotop production}

In the second class of scenarios implying the production of monotop states, the top quark is produced in association with an invisible bosonic field that couples in a flavour-changing 
way to top and light up-type (up or charm) quarks. The bosonic state is in general not stable since it couples to quarks. A missing energy signature is therefore enforced by requiring these fields either to be long-lived so that they decay outside of the detector, or to decay predominantly into a pair of additional neutral stable particles. In particular, the latter possibility has been proposed in the framework of flavourful dark matter models [9], where the extra boson $\left(\phi\right.$ or $\left.V_{\mu}\right)$ is a mediator of the interactions of the dark matter candidate with the Standard Model particles.

The main issue with this class of models is to ensure that the new boson leads to a missing energy signature in a detector. In this work, we address it by assuming that the $\phi / V$ field dominantly decays into a pair of dark matter candidate particles. ${ }^{1}$ In this case, extra constraints arise from the requirement that the particle the boson decays into is a good candidate for dark matter, or that at least it does not overpopulate the Universe.

Spin-0 mediator. As already stated in section 2.1, the interactions of a scalar field to quarks involve both the right-handed and left-handed components of the fermions. Consequently, the scalar $\phi$ field must transform as a doublet of $\mathrm{SU}(2)_{L}$ with an hypercharge quantum number of $1 / 2$,

$$
\phi=(\underset{\sim}{\mathbf{1}}, \underset{\sim}{\mathbf{2}}, 1 / 2) \equiv\left(\begin{array}{c}
\phi^{+} \\
\phi^{0}
\end{array}\right) .
$$

The coupling in the effective Lagrangian can be written as

$$
y^{i j} \phi \bar{q}_{L, i} u_{R, j}+\text { h.c. }
$$

where $i, j$ span over the quark flavours. The new scalar has the same quantum numbers as the Brout-Englert-Higgs field, thus one can also write a coupling to the right-handed down-type quarks and mix $\phi$ with the Higgs with the potential harm of generating a nonvanishing vacuum expectation value. The presence alone of couplings to both up-type and down-type quarks already generates dangerous flavour-changing effects. Nevertheless, we assume, in a first step, that the only extra coupling with respect to the Standard Model is the one of eq. (2.18) and will show, in the following, that it is already hard to construct a phenomenologically viable model. Gauge invariance implies the presence of interactions between the charged component field $\phi^{+}$and quarks, so that the $\phi^{+}$field always promptly decays into two-body final states, $\phi^{+} \rightarrow u \bar{b}$ or $t \bar{d}$. Analogously, the neutral component $\phi^{0}$ could also decay into an associated particle pair comprised of a top and an up quark, $\phi^{0} \rightarrow u \bar{t}+t \bar{u}$, as well as into a three-body final state via the exchange of a virtual charged scalar field, ${ }^{2} \phi^{0} \rightarrow W^{-}\left[\phi^{+}\right]^{*}+W^{+}\left[\phi^{-}\right]^{*} \rightarrow W^{-} \bar{b} u+W^{+} b \bar{u}$ or $W^{-} \bar{d} t+W^{+} d \bar{t}$. All these decay channels are however assumed to be negligible when compared to a decay into a pair of dark matter particles. In this case, no minimal coupling to a single stable state is achievable since $\phi$ is a doublet of $\mathrm{SU}(2)_{L}$, and one must design an interaction of the $\phi$ state

\footnotetext{
${ }^{1}$ One could also consider that neither the boson nor its decay products are stable, but instead long-lived. Although this is a viable assumption, this implies further complications in the building of the model. We therefore stick with the minimal case.

${ }^{2}$ Due to reasons already stated in section 2.1 , the $\phi^{+}$and $\phi^{0}$ states are assumed to have similar masses.
} 
to two extra fields whose combination forms a doublet of $\mathrm{SU}(2)_{L}$. If we restrict ourselves to $\phi^{0}$-decays into fermionic particles, the most minimal option is given by the Lagrangian

$$
\mathcal{L}_{\phi-\text { decay }}=y_{\chi} \phi \bar{\chi}_{d} \chi_{s}+\text { h.c. },
$$

where $\chi_{s}$ is an electroweak singlet and $\chi_{d}$ a weak doublet with an hypercharge of $1 / 2$. This term induces decays of both components of $\phi$

$$
\phi^{0} \rightarrow \chi_{s} \chi_{d}^{0} \quad \text { and } \quad \phi^{+} \rightarrow \chi_{d}^{+} \chi_{s} \rightarrow\left[W^{+}\right]^{*} \chi_{d}^{0} \chi_{s},
$$

the charged component $\chi_{d}^{+}$being taken heavier than, but close in mass to, the neutral component $\chi_{d}^{0}$ so that both neutral fields $\chi_{s}$ and $\chi_{d}$ can be seen as viable dark matter candidates.

As a consequence of this non-minimal dark sector of the model, monotop production via flavour-changing interactions of up-type quarks with a new invisible scalar field will always be accompanied by an extra single top production mode

$$
p p \rightarrow t \phi^{-} \rightarrow t \chi_{d}^{0} \chi_{s}^{0}\left[W^{-}\right]^{*} .
$$

The nature and magnitude of the associated effects are very benchmark dependent. For instance, a small mass splitting between the component fields of $\chi$ leads to very soft $W$ boson decay products, so that the process of eq. (2.21) would imply new contributions to monotop production. On the other hand, in the case of larger mass splittings, related new physics scenarios feature an LHC signature comprised of a single top quark and an isolated lepton.

Nevertheless, we choose to keep the focus on minimal models, and therefore ignore, in the rest of this work, scenarios where monotop states are produced from flavour-changing interactions of up-type quarks with a scalar particle mediating dark matter couplings to the Standard Model.

Spin-1 mediator. When the mediator is a vector boson $V$, one can design very simple models since it can be singlet under the electroweak group. In this setup, the associated couplings involve either right-handed or left-handed quarks and take the form

$$
\left(a_{R}^{i j} V_{\mu} \bar{u}_{R, i} \gamma^{\mu} u_{R, j}+a_{L}^{i j} V_{\mu}\left(\bar{u}_{L, i} \gamma^{\mu} u_{L, j}+\bar{d}_{L, i} \gamma^{\mu} d_{L, j}\right)+\text { h.c. }\right),
$$

where the $a_{L, R}$ parameters denote the strengths of the interactions of the $V$-field with the quarks. As in the rest of this section, we restrict ourselves to interactions focusing on the monotop hadroproduction modes, so that only couplings involving the third generation are assumed to be present. Moreover, our analysis will focus on monotop production modes enhanced by parton densities. As a consequence, interactions to second generation quarks are ignored. Furthermore, being a singlet, $V$ can mix via a kinetic term with the hypercharge gauge boson in the Standard Model: such mixing will in turn generate couplings of $V$ with all the quarks and leptons proportionally to their hypercharge. As a result, bounds on the new particle $V$ can be derived from many new production and decay 
processes, like for intance, its Drell-Yan production followed by a dilepton decay. For this reason, we ignore possible kinetic mixing of the $V$ field in the following.

The Lagrangian terms of eq. (2.22) open various decay channels for the $V$-field. Recalling that only couplings involving the first and third generation quarks have been retained, non-vanishing left-handed couplings allow the mediator to promptly decay into jets initiated by down-type quarks, $V \rightarrow b \bar{d}+d \bar{b}$. Next, the importance of the decays into top and up quarks (this time both in the context of left-handed and right-handed couplings) depends on the mass hierarchy between the mediator and the top quark, the tree-level decay $V \rightarrow t \bar{u}+u \bar{t}$ being only allowed when $m_{V}>m_{t}$. Furthermore, when $m_{V}<m_{t}$, a triangle loop-diagram involving a $W$-boson could also contribute to the decay of the $V$-field into a pair of jets, $V \rightarrow d_{i} \bar{d}_{j}$. Finally, when $m_{W}<m_{V}<m_{t}$, the three-body decay channel $V \rightarrow b W^{+} \bar{u}+\bar{b} W^{-} u$ is open, mediated by a virtual top quark. A monotop signal is thus expected only when the $V$-field is invisible and dominantly decays into a pair of dark matter particles. Since $V$ is an electroweak singlet, the associated couplings can be written, in the case of fermionic dark matter, as

$$
\mathcal{L}_{V \text {-decay }}=V_{\mu}\left(g_{R \chi} \bar{\chi}_{R} \gamma^{\mu} \chi_{R}+g_{L \chi} \bar{\chi}_{L} \gamma^{\mu} \chi_{L}\right),
$$

where $\chi$ is a Dirac fermion, singlet under the Standard Model gauge symmetries. The consistency of the model, i.e., the requirement that $V$ always mainly decays into a pair of $\chi$-fields and not into one of the above-mentioned visible decay modes, implies constraints on the Lagrangian parameters. They will be studied in details in the next section, together with other requirements that can be applied to viable non-resonant monotop scenarios.

For completeness, one can also couple the $V$-boson to left-handed quarks in (nonminimal) scenarios where it lies in a triplet of $\mathrm{SU}(2)_{L}, V_{t}=(\underset{\sim}{\mathbf{1}}, \underset{\sim}{\mathbf{3}}, 0)$. In this case, it is however difficult to build couplings to a minimal dark matter sector, the simplest case being the one of a fermionic doublet of $\mathrm{SU}(2)_{L}$. This also predicts the existence of a charged component that can be produced, at the LHC, in association with a single top quark or that can give rise to a monobottom signature (for small mass gaps among the vector degrees of freedom). Following the minimality principle, we will not consider this case any further.

Summary for the non-resonant channel. Summarising all the considerations above, the minimal gauge-invariant Lagrangian yielding monotop production in the flavourchanging mode is given by

$$
\begin{aligned}
& \mathcal{L}_{\text {non-res }}=\mathcal{L}_{\text {kin }}(V, \chi)+V_{\mu}\left(g_{R \chi} \bar{\chi}_{R} \gamma^{\mu} \chi_{R}+g_{L \chi} \bar{\chi}_{L} \gamma^{\mu} \chi_{L}\right) \\
&+\left(a_{R}^{i j} V_{\mu} \bar{u}_{R, i} \gamma^{\mu} u_{R, j}+a_{L}^{i j} V_{\mu}\left(\bar{u}_{L, i} \gamma^{\mu} u_{L, j}+\bar{d}_{L, i} \gamma^{\mu} d_{L, j}\right)+\text { h.c. }\right),
\end{aligned}
$$

where the first term contains kinetic, mass and gauge interaction terms for the $V$ and $\chi$ fields. In the notations of refs. $[8,15]$, the second line of the Lagrangian reads

$$
\mathcal{L}_{\text {non-res }}=\mathcal{L}_{\text {kin }}+\left(V_{\mu} \bar{u}_{i}\left[\left(a_{F C}^{1}\right)^{i j} \gamma^{\mu}+\left(b_{F C}^{1}\right)^{i j} \gamma^{\mu} \gamma^{5}\right] u_{j}+\text { h.c. }\right),
$$

so that the two parameterizations are related by

$$
a_{F C}^{1}=\frac{a_{R}+a_{L}}{2}, \quad b_{F C}^{1}=\frac{a_{R}-a_{L}}{2} .
$$


The two parameter bases are therefore equivalent, although gauge invariance imposes that non-vanishing left-handed couplings relevant for monotop production are accompanied by interactions with left-handed down-type quarks too. Since such interactions also enable the production of mono $(b$-)jet final states, a full analysis of this scenario should account for monojet search results. In the following, we will mainly focus on the case $a_{L}=0$ unless specified. Note also that in general $\chi$ may be a Majorana fermion, however this is a less likely situation as, in order to couple to $V$, the dark matter candidate is expected to carry a U(1) charge. In the following, we limit ourselves to the Dirac case, but the results in the Majorana case are qualitatively similar.

\section{Monotop phenomenology specific to non-resonant models}

Some features of the resonant models mediated by a scalar, like the lifetime of the invisible fermion produced in association with the top quark, have been studied in details in ref. [11]. In the following, we therefore focus on various features of non-resonant spin- 1 models by studying the effective lifetime of the invisible vector, associated single top signals, and the dark matter relic density. We separately consider two regions of the parameter space which have very different phenomenology: the case where the mediator is lighter than the top quark (its mass $m_{V}$ being smaller than the top mass $m_{t}$ ) and the case where it is heavier, with $m_{V}>m_{t}$. On the basis of the minimality argument employed in the previous section, we also restrict ourselves to the case where monotop production is the only expected new physics signature of the model. We therefore set $a_{L}=0$ in the effective Lagrangian of eq. (2.24). As stated above, non-vanishing $a_{L}$ values imply mono(b-)jets production, and the associated constraints may be predominant. The corresponding detailed study is postponed to future work.

\subsection{Mediators heavier than the top quark}

We first start with the scenario of heavy mediators: the mediator $V$ is not long lived as it can always decay into a top quark. Including in the model a $V$-decay channel into an invisible state to be considered as a dark matter candidate is thus always necessary. Focusing on the minimal case, we study below the interesting interplays between the requirement that the invisible channel dominates and bounds originating from the relic density of the dark matter candidate.

\subsubsection{Tree-level decays of the mediator}

When the $V$-boson is heavier than the top quark, it can decay into either a pair of downtype quarks, an associated pair comprised of a top quark and a lighter quark or a pair of dark matter particles, as already discussed in section 2.2. Since the first two decay modes are driven by the same interaction vertices allowing one for monotop production, we need to make sure that the invisible decay channel always dominates. As we focus on the couplings to up and top quarks, we set all the couplings $a_{R, L}^{i j}=0$ except for $a_{R, L}^{13}$ and 
$a_{R, L}^{31}$. The relevant partial widths are given by ${ }^{3}$

$$
\begin{aligned}
\Gamma(V \rightarrow b \bar{d}+\bar{b} d) & =\frac{m_{V}}{4 \pi}\left|a_{L}^{31}+a_{L}^{13, *}\right|^{2} \\
\Gamma(V \rightarrow t \bar{u}+\bar{t} u) & =\frac{m_{V}}{4 \pi}\left|a_{R}^{31}+a_{R}^{13, *}\right|^{2}\left(1-\frac{m_{t}^{2}}{m_{V}^{2}}\right)\left(1-\frac{m_{t}^{2}}{2 m_{V}^{2}}-\frac{m_{t}^{4}}{2 m_{V}^{4}}\right), \\
\Gamma(V \rightarrow \chi \chi) & =\frac{m_{V}}{24 \pi} \sqrt{1-4 \frac{m_{\chi}^{2}}{m_{V}^{2}}}\left[\left(\left|g_{L \chi}\right|^{2}+\left|g_{R \chi}\right|^{2}\right)\left(1-\frac{m_{\chi}^{2}}{m_{V}^{2}}\right)+\frac{6 m_{\chi}^{2}}{m_{V}^{2}} \Re\left\{g_{L \chi} g_{R \chi}^{*}\right\}\right],
\end{aligned}
$$

where we neglect all quark masses but the top mass. In addition, we denote by $m_{\chi}$ the mass of the dark matter candidate. In the minimal scenario where only right-handed couplings are present, $a_{L}=0$, the decay to light quarks vanishes and we are left with two decay channels above the top threshold. For future convenience, we define $a_{R}=a_{R}^{31}+a_{R}^{31, *}$.

In this set-up, we study typical constraints that can be imposed on ratios of the $g_{L \chi}$, $g_{R \chi}$ and $a_{R}$ parameters when they are all assumed to be real quantities. Since ratios of branching ratios are equivalent to ratios of partial widths, we use this latter quantity and show, in figure 1, the maximum value of the $a_{R}$ coupling strength in units of the $\chi V$ coupling that ensures the $V$-field to decay invisibly in at least $99 \%$ of the cases. In the left panel of the figure, we consider scenarios where $g_{R \chi}$ vanishes (the same result holds for vanishing $g_{L \chi}$ ), while in the right panel of the figure, we assume vector-like couplings, $g_{L \chi}=g_{R \chi}=g_{V \chi}$. In general, the coupling to the top quark $a_{R}$ (that is responsible for the monotop signal) has to be quite small compared to the coupling to the dark matter candidate in order for the mediator $V$ to be invisible, unless the mass of the mediator $V$ is close to the top mass. On the contrary, if the mass of $V$ is close to the $\chi \chi$ threshold, the invisible decay modes are suppressed. This study shows that it is not straightforward to have $V$ to decay invisibly, and this constraint may play an important role in the interpretation of the signal, especially when associated with the study of the properties of $\chi$ as a dark matter candidate. We study more in detail this question in the next subsection.

Similar conclusions would hold in less minimal models, like the one with a left-handed coupling $a_{L}$ where the decay to down-type quarks is open and dominant also below the top threshold.

\subsubsection{Dark matter constraints}

We have seen that, in order to avoid visible decays of the mediator $V$, it has to be coupled to a stable particle $\chi$ and the decay $V \rightarrow \chi \chi$ must always dominate. If $\chi$ is stable, and if the model is minimal in the sense that $V$ is the only mediator of interactions between the dark sector and the Standard Model, then the only annihilation process that will determine the thermal relic abundance of $\chi$ is $\chi \chi \rightarrow V \rightarrow t \bar{u}$ and $\bar{t} u$. Such process is proportional to the same coupling that gives rise to the monotop signature at the LHC, and also to the coupling of $V$ to dark matter. By studying the relic abundance of $\chi$ one can therefore derive interesting constraints on the couplings, especially when imposing that the relic abundance is smaller than the measured density of dark matter. Those restrictions can in

\footnotetext{
${ }^{3}$ The results have been checked using the decay module of FEYNRules [28].
} 

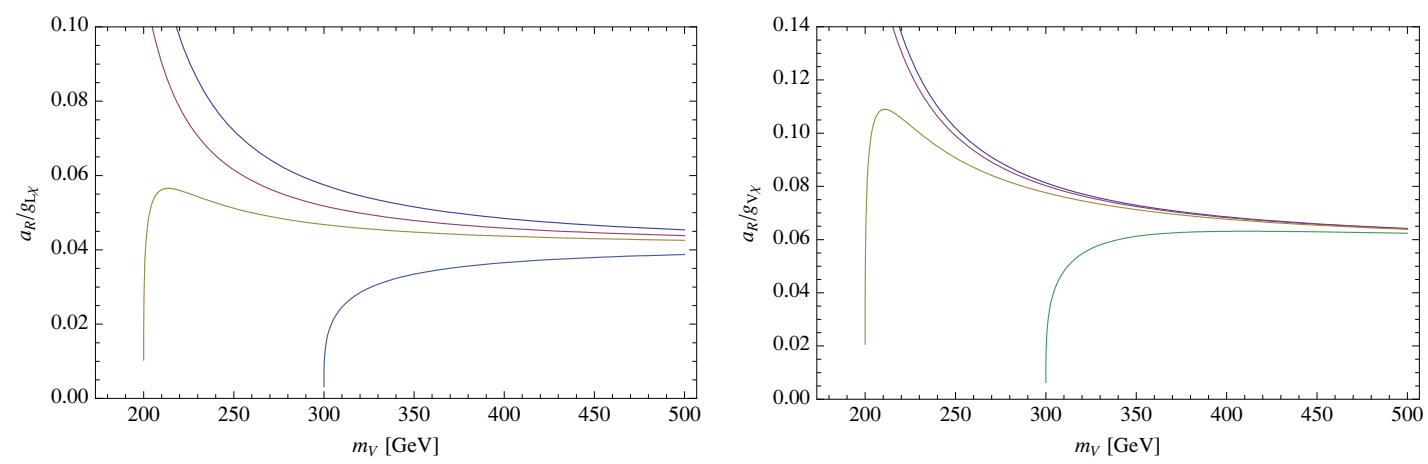

Figure 1. Maximum value of $a_{R}$ necessary to enforce the mediator $V$ to decay invisibly in $99 \%$ of the cases. We focus on scenarios where the couplings of the mediator to dark matter are chiral with $g_{R \chi}=0$ (or $g_{L \chi}=0$ ) in the left panel, and vector with $g_{L \chi}=g_{R \chi}=g_{V \chi}$ in the right panel. The four curves correspond to $m_{\chi}=5,75,100$ and $150 \mathrm{GeV}$ from the lower to the upper ones in each figure.

principle always be evaded by assuming that there are additional mediators, or that $\chi$ is not a stable particle but rather a long-lived one that decays on cosmological time scales. In the rest of the section, we nevertheless focus on the minimal case of $\chi$ being the only dark matter candidate.

As the relic abundance decreases with increasing annihilation cross sections, one can calculate a lower bound on the product of $a_{R}$ with the couplings of $V$ to the dark matter by requiring that the relic abundance is equal or smaller than the measured one. Values of the couplings below the bound would be excluded as the stable particle would overpopulate the Universe. The bound has been computed by implementing the model described by the Lagrangian of eq. (2.24) in CALCHeP [29]. For the calculation of the relic abundance, we used the usual approximate formulas deriving from an analytic solution of the Boltzmann equation (see ref. [30] for more details):

$$
\Omega_{D M} h^{2}=\frac{1.04 \cdot 10^{9}}{M_{P l}} \frac{x_{F}}{\sqrt{g_{*}}} \frac{1}{\langle\sigma v\rangle}
$$

where $x_{F}=m_{\chi} / T_{F}$ and the freeze-out temperature is $T_{F} \sim 25 \mathrm{GeV}, g_{*}=92$ is the number of relativistic degrees of freedom at freeze-out, and all dimensionful quantities are in $\mathrm{GeV}$. We consider, for concreteness, a vectorial model with $g_{L \chi}=g_{R \chi}=g_{V \chi}$. The results of the calculation are shown in figure 2, where we present the lower bound on $a_{R} \times g_{V \chi}$ as a function of the mediator mass $m_{V}$ and the dark matter mass $m_{\chi}$. We restrict ourselves to values of the $\chi$ mass above the top threshold, $2 m_{\chi}>m_{t}$, so that a two-body process is kinematically allowed. Below the top threshold, the dark matter candidate can only annihilate into threebody final states or via loop-induced processes, so that the annihilation cross section is too small and the $\chi$ particle overpopulates the Universe. The figure shows that the product of couplings is bound to be larger than about 0.1 , with the lower bound increasing towards the top threshold as the phase space closes down, and becomes smaller towards the $V$ threshold $2 m_{\chi}=m_{V}$ where the resonant $V$ exchange enhances the annihilation. We recall that the $V$-boson mass must be at least twice as large as the dark matter candidate mass to 


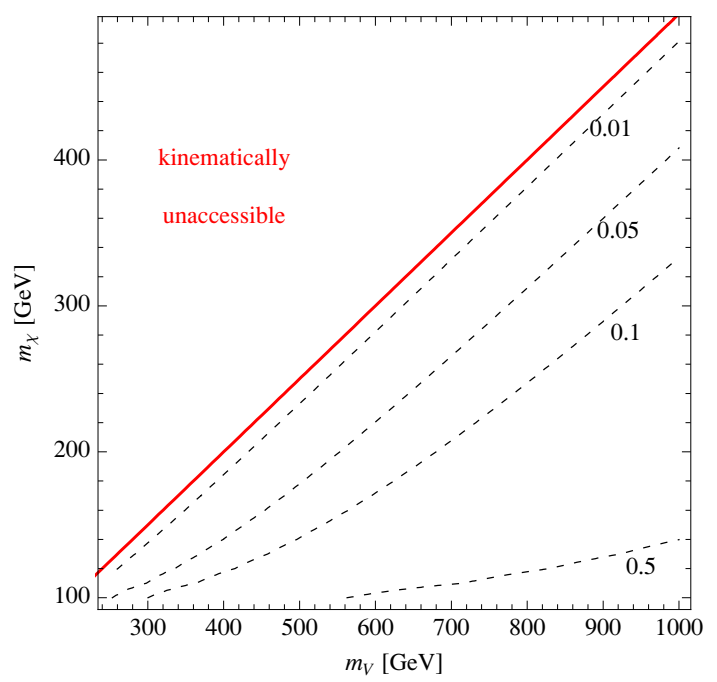

Figure 2. Lower bound on $g_{V \chi} \times a_{R}$ from the dark matter relic abundance as a function of $m_{V}$ and $m_{\chi}$.

allow invisible decays for $V$. The corresponding regions of the parameter space are tagged as kinematically inaccessible.

This result, very interesting per se, can be combined with other constraints to better determine the viable regions of the parameter space of the model. The requirement that the invisible $V$-decay dominates has allowed us, in section 3.1.1, to calculate a lower bound on the ratio $g_{V \chi} / a_{R}$ which depends on the mediator and dark matter masses (see figure 1). Multiplying it with the limits derived from the relic abundance predictions, we extract a lower bound on $g_{V \chi}$ independently of the value of $a_{R}$ : the results are shown in figure 3. The lower bound on $g_{V \chi}$ is found to grow with smaller values of the $\chi$ mass. Moreover, near the top threshold, it reaches values well above unity, tending hence to the non-perturbative regime.

Under the assumption that $\chi$ is the only dark matter candidate of the theory, we can further restrict our analysis to parameter space regions where the values of the couplings are such that the bound from the dark matter abundance is saturated. We first reinterpret, as a function of the masses, the limits calculated in the CMS monotop search [19] by accounting for an invisible branching ratio of the mediator that may not be $100 \%$. Next, we correlate these to the dark matter results: for increasing values of $a_{R}$, the coupling $g_{V \chi}$ has to be smaller to satisfy the dark matter constraints. This indicates that an enhancement of the $t V$ production rate (by increasing $a_{R}$ ) is accompanied by a reduction of the invisible branching ratio of $V$, which possibly reduces the production cross section of monotop systems.

A general bound on $a_{R}$ can be obtained using the relation

$$
a_{R}^{2} \times \frac{k^{2} / a_{R}^{2} \tilde{\Gamma}_{\chi \chi}}{k^{2} / a_{R}^{2} \tilde{\Gamma}_{\chi \chi}+a_{R}^{2} \tilde{\Gamma}_{t u}} \leq a_{R-C M S}^{2}
$$

where $\tilde{\Gamma}$ denote the partial widths into $\chi \chi$ and $t u$ final states given by eq. (3.1) stripped by the coupling strengths, $a_{R-C M S}$ is the upper bound on $a_{R}$ derived from the CMS analysis 


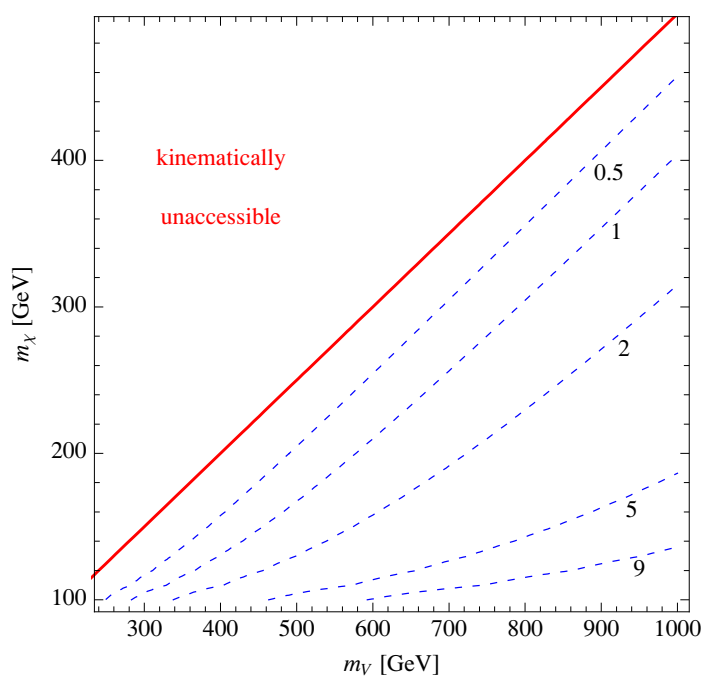

Figure 3. Lower bound on $g_{V \chi}$ obtained combining the dark matter relic abundance constraints with the requirement that the mediator $V$ decays invisibly in $99 \%$ of the cases.

that assumes that $V$ decays are always invisible, and $k$ is the lower bound on $g_{V \chi} \times a_{R}$ deduced from the dark matter relic abundance in figure 2. On the left panel of figure 4, we extract the bound on $a_{R-C M S}$ from the CMS analysis of ref. [19]. Inverting the above equation, bounds on $a_{R}$ for a $\chi$ particle saturating the dark matter relic abundance can then be rewritten as

$$
\begin{aligned}
a_{R}^{2} & \leq \frac{k^{2} \tilde{\Gamma}_{\chi \chi}}{2 a_{R-C M S}^{2} \tilde{\Gamma}_{t u}}\left(1-\sqrt{1-4 \frac{a_{R-C M S}^{4} \tilde{\Gamma}_{t u}}{k^{2} \tilde{\Gamma}_{\chi \chi}}}\right), \\
\text { or } \quad a_{R}^{2} & \geq \frac{k^{2} \tilde{\Gamma}_{\chi \chi}}{2 a_{R-C M S}^{2} \tilde{\Gamma}_{t u}}\left(1+\sqrt{1-4 \frac{a_{R-C M S}^{4} \tilde{\Gamma}_{t u}}{k^{2} \tilde{\Gamma}_{\chi \chi}}}\right) .
\end{aligned}
$$

The result is shown on the right panel of figure 4. Above the blue curve, the argument of the square root is negative and the inequalities of eq. (3.4) have no solution, therefore there is no bound that can be applied on $a_{R}$. Below the blue line, near the top threshold, the dark matter constraint requires larger couplings and therefore larger monotop rates are allowed, thus a bound on $a_{R}$ can be calculated. Naturally, larger portions of the parameter space are expected to be covered with the upcoming run II of the LHC.

The region where the monotop signal is suppressed can have interesting additional features. The boson $V$ may dominantly decay into top and lighter quarks, yielding at the same time a signature comprised of same-sign top quark pairs $(t V \rightarrow t t \bar{u})$ and extra contributions to top-antitop production $(t V \rightarrow t \bar{t} u)$ that may be difficult to observe due to the overwhelming $t \bar{t}$ Standard Model background. These extra channels deserve a particular attention, in particular in upcoming data from LHC collisions at $\sqrt{s}=13 \mathrm{TeV}$.

\subsection{Mediators lighter than the top quark}

When the spin-1 mediator $V$ is lighter than the top quark, its possible decay modes into a top and a lighter quark are kinematically forbidden. At tree-level, in the minimal scenario 

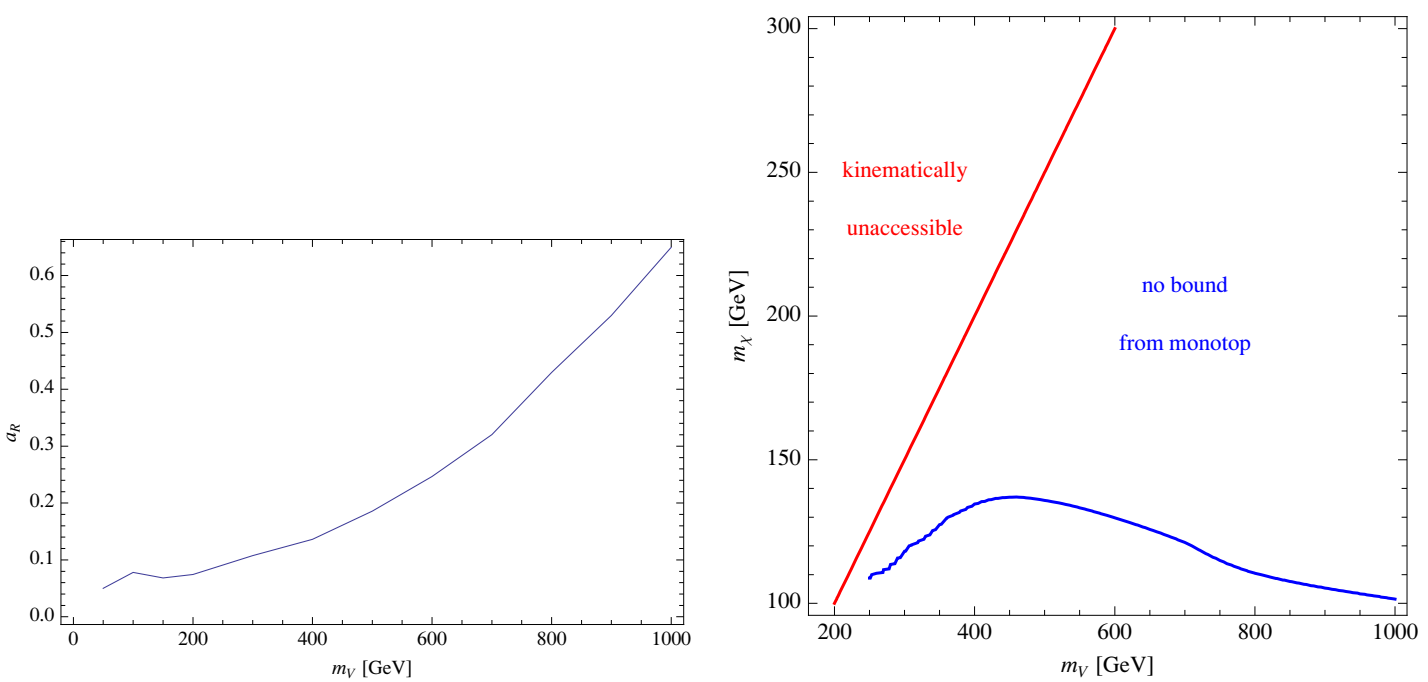

Figure 4. Reinterpretation of the CMS monotop limits of ref. [19] in terms of $a_{R}$ (left) for a $100 \%$ invisible mediator. The results are then used to determine the viable regions of the parameter space when enforcing dark matter and LHC constraints as shown by eq. (3.4) (right). The region above the blue line is found not to be bounded by current searches. Below the blue line, limits on $a_{R}$ deduced from the left panel of the figure are in order.

$a_{L}=0, V$ can therefore only decay into a multibody final state such as $V \rightarrow u \bar{b} W^{-}$or $\bar{u} b W^{+}$, where the $W$-boson is virtual when $m_{V}<m_{W}$ ( $m_{W}$ denoting the $W$-boson mass). ${ }^{4}$ In this mass range, loop-induced decays must however be considered too. For instance, a triangle loop-diagram with a $W$-boson exchange generates couplings to down-type quarks, thus opening a dijet decay channel. As the decay channels in this region are either kinematically or loop-suppressed, one may wonder whether $V$ may be long-lived without the need for an additional invisible decay channel. Another interesting property of this mass region is that a new decay of the top quark is allowed, $t \rightarrow u V$, and extra constraints on monotop scenarios could therefore be extracted from, e.g., top width measurements or the analysis of $t \bar{t}$ events when one of the top quarks decays into a jet plus missing energy.

\subsubsection{Loop-induced and multibody decays of the mediator}

Light mediators, below the top mass threshold, may decay dominantly into two jets via loop-induced interactions. The structure of the loop crucially depends on the chirality of the monotop couplings: for instance, in the case $a_{L} \neq 0$, the loop is divergent, which signals that a tree-level coupling to down-type quarks is necessary for the theory to be consistently renormalizable. This result confirms the necessity to consider an effective model fully invariant under the electroweak symmetry.

In the minimal case $a_{L}=0$, the loop contributions turn out to be finite. Since weak interactions are left-handed, the chiralities of the quarks involved in these diagrams must be flipped, which implies that the loop-induced couplings are proportional to the product of the up and top masses $m_{u} m_{t}$. Contrary to setups where monotops are produced

\footnotetext{
${ }^{4}$ When $a_{L} \neq 0$, the decays into a pair of down quark will always dominate.
} 

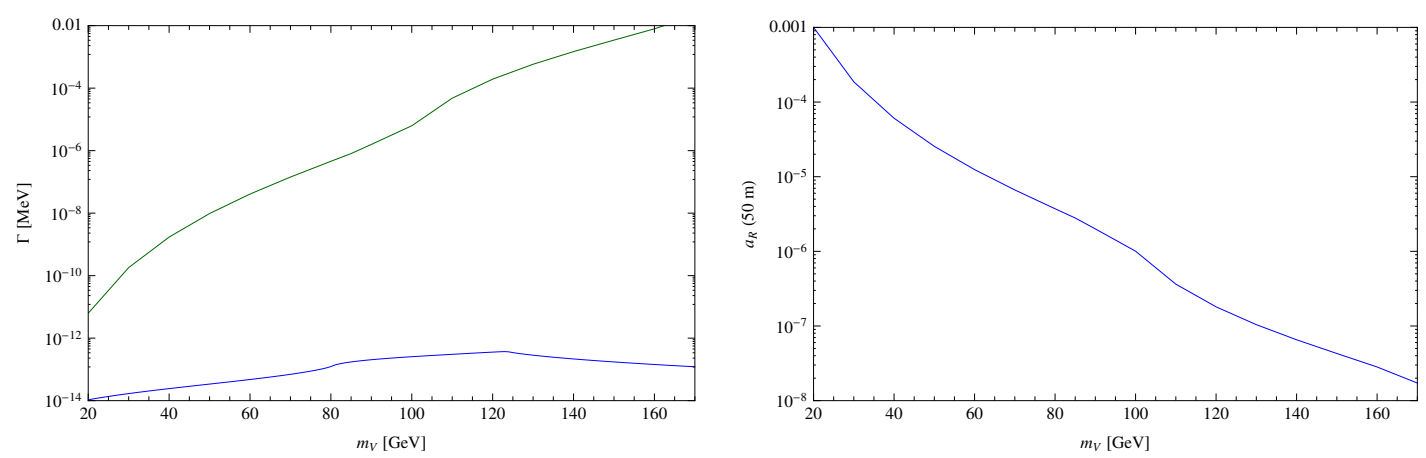

Figure 5. Partial decay width associated with the loop-induced (blue) and multibody (green) decay of the $V$-field into down-type quarks as a function of the $V$-boson mass, as given by eq. (3.7) and returned by MADWIDTH (left panel). We consider scenarios in which $a_{R}=0.04$ and $m_{V}$ is kept smaller than the top mass. The results are translated, in the right panel, as a bound on $a_{R}$ that ensures that the $V$-boson has a decay length of at least $50 \mathrm{~m}$.

from left-handed interactions of the mediator with quarks, the loop-induced $V d_{L} d_{L}$ couplings are thus finite, in line with the fact that no associated counterterm appears after renormalization. The interaction strength reads, in the limit of small light quark masses,

$$
g_{V d^{i} d^{j}}^{1-\mathrm{lop}}\left(a_{R}\right)=\frac{\alpha a_{R}}{4 \pi s_{W}^{2}} \frac{m_{u}}{m_{t}}\left(V_{u d^{i}}^{*} V_{t d^{j}}+V_{t d^{i}}^{*} V_{u d^{j}}\right) \tilde{c}_{0},
$$

where $\alpha$ stands for the electromagnetic coupling constant, $s_{W}$ for the sine of the weak mixing angle and $V_{i j}$ for the elements of the CKM matrix. In addition, the loop factor

$$
\tilde{c}_{0}=m_{t}^{2} C_{0}\left(p_{1},-\left(p_{1}+p_{2}\right) ; m_{W}, m_{t}, 0\right)
$$

depends on the Passarino-Veltman three-point function $C_{0}$ where $p_{1}$ and $p_{2}$ are the momenta of the external down-type quarks. We can therefore calculate the partial width associated with the decay $V \rightarrow \bar{d}^{i} d^{j}$ which reads, after summing over all down-type quark flavours,

$$
\Gamma(V \rightarrow j j)=\frac{\alpha^{2} a_{R}^{2}}{64 \pi^{3} s_{W}^{4}} \frac{m_{V} m_{u}^{2}}{m_{t}^{2}}\left|\tilde{c}_{0}\right|^{2}
$$

We observe that it exhibits both a loop-suppression and a $\left(m_{u} / m_{t}\right)^{2}$ factor, so that it is expected to be numerically small.

In figure 5, we show the partial width in eq. (3.7) as a function of the mediator mass for $a_{R}=0.04$ (left panel, blue curve). We compare this result to preditions for three-body and four-body decays (left panel, green) as calculated by MADWIDTH [28], which turn out to be dominant upon the entire mass range. On the right panel of the figure, the partial width is translated as an upper bound on the value of $a_{R}$ in order for $V$ to have a mean decay length of at least 50 metres so that it is long-lived enough to decay outside of typical hadron collider detectors. The figure shows that the lifetime of $V$ would be long enough only for extremely small values of the coupling $a_{R}$ that will challenge the possible observation of a monotop signal at the LHC by reducing the associated production cross section. The only 


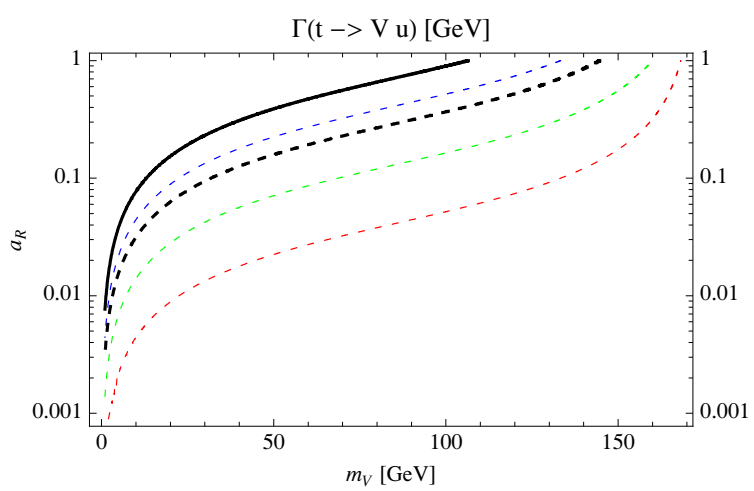

Figure 6. Partial width associated with the $t \rightarrow V u$ decay mode of the top quark as a function of the $V$-boson mass and the $a_{R}$ coupling. We show curves for a partial width of $3,1,0.5,0.1$ and $0.01 \mathrm{GeV}$. The solid black curve corresponds to the upper bound on $a_{R}$ from a partial width less than $3 \mathrm{GeV}$, roughly corresponding to the direct measurement in ref. [31].

way out is thus to extent the theoretical framework so that invisible decay channels are enabled, as in the previous section. It should also be mentioned that above the $W$-boson threshold, a tree-level three-body decay is kinematically open, which further shortens the decay length of $V$. Finally, in cases where the model features a coupling of the $V$-field to top and charm quarks, the partial width of eq. (3.7) would exhibit an enhancement proportional to $\left(m_{c} / m_{u}\right)^{2}$.

In summary even for monotop scenarios in which the mediator cannot decay into a top quark, its lifetime is generally too short and one needs to complete the model by adding a decay channel into an invisible state. Although the class of minimal scenarios described in this section features a light extra vector boson, the setup is compatible with current Tevatron and LHC bounds on monotop production as the latter are always derived under the assumption of very large coupling values of $\mathcal{O}(0.1)[18,19]$. They could however be constrained by other observations, as will be shown in the next subsections.

\subsubsection{Single top constraints on monotop scenarios}

Motivated by minimality principles, we have discussed, in the previous section, appealing monotop scenarios in which the mediator $V$ is lighter than the top quark. In this case, the former couples to up and top quarks via right-handed couplings and one needs to add an invisible decay channel to potential dark matter particles $\chi$ to guarantee a monotop signature, unless the coupling strength $a_{R}$ is very small. On different grounds, these scenarios feature a new decay channel for the top quark, $t \rightarrow u V$. This observation can be used to further restrict the viable regions of the parameter space by imposing that new physics contributions to the top width do not challenge the measured value of $1.10<\Gamma_{t}<4.05 \mathrm{GeV}$ [31], from direct measurements at $\mathrm{CDF}$. A more precise measurement, which takes the value $\Gamma_{t}=2.0 \pm 0.5 \mathrm{GeV}[32]$, can also be obtained by fitting the single-top measurements. However, the latter does not apply in our scenario where new physics contributions to single-top can arise. Assuming a good agreement between the Standard Model expectation and the top width measurement, therefore, the partial width $\Gamma(t \rightarrow V u)$ can thus be enforced to 
be of at most $3 \mathrm{GeV}$. On figure 6 , we present the dependence of this partial width on the coupling $a_{R}$ and the mediator mass $m_{V}$. We observe that for couplings smaller than 0.01 , new physics effects in the top width are predicted to be very small, except when the mediator is almost massless. This consequently disfavours such setups in which the mediator is very light, even in cases with coupling strengths of $\mathcal{O}(0.001)$.

Kinematically allowed $t \rightarrow V u$ decays also imply that monotop events can be issued from the production of a top-antitop pair when one of the top quarks decays into a $V$-boson and a light quark,

$$
p p \rightarrow t \bar{t} \rightarrow t \bar{u} V \quad \text { or } \quad p p \rightarrow t \bar{t} \rightarrow \bar{t} u V
$$

This process induces additional contributions to the production of a monotop system $(t V$ or $\bar{t} V$ ) in association with an additional jet, a signature already accounted for in the LHC monotop analysis of ref. [19]. How much this new channel will contribute to the monotop signal depends on the cuts employed in the experimental analysis. However, due to the large $t \bar{t}$ cross section, these effects cannot be neglected.

Complementary constraints on this channel could be deduced from Standard Model single top analyses whose signal regions could capture monotop events as above. For instance, both CMS [33] and ATLAS [34] have analyses dedicated to the measurement of the single top cross section in the $t$-channel which contain a region that could be populated by monotop events as above. ${ }^{5}$ In the CMS analysis, events are selected by requiring one single isolated electron or muon and exactly two jets, one of them being $b$-tagged. The background is reduced by requiring an important amount of missing energy and by imposing that the transverse mass computed after combining the lepton transverse momentum with the missing transverse momentum is large. A final selection is preformed by means of an advanced multivariate technique. We have nevertheless to ignore this last step of the selection as the amount of information provided in the experimental publication is not sufficient for satisfactorily recasting it (see ref. [35] for more information on this aspect).

We simulate our new physics signal by using the monotop model [8] implemented in the FEYNRules package [36, 37], tuning the model parameters to the setup of eq. (2.24), so that we can export the model to a UFO library [38] that is then linked to MADGraph5_AMC@NLO [39]. The generated parton-level events have subsequently been processed by PYThia [40] for parton showering and hadronization and by DeLPHES [41] for detector simulation, making use of the recent 'MA5Tune' [42] of the CMS detector description of DeLPhes. The CMS analysis of ref. [33] has finally been implemented in the MADANALYsis5 framework [43, 44], which has allowed us to derive exclusion bounds at the $95 \%$ confidence level in the $\left(m_{V}, a_{R}\right)$ plane, as shown on figure 7 . The figure also shows the constraint from the top width, and from the dedicated CMs monotop search [19]. The monotop search is currently more sensitive. However, the bound from the single top is a rough estimate, and the bound may be much stronger once the full analysis, including the multivariate selection, is taken into account. Nevertheless, our result shows that the

\footnotetext{
${ }^{5}$ Other single top analyses could be considered. However, they in general use multivariate techniques that cannot be employed in the reinterpretation framework pursued below.
} 


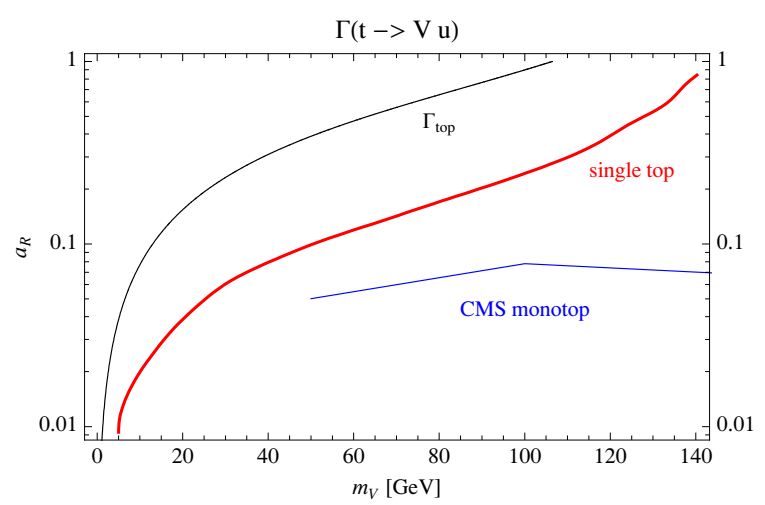

Figure 7. Parameter space region excluded (the area above the red curve) at the $95 \%$ confidence level by the CMS single top analysis of ref. [33] before the multivariate selection, compared to the exclusion from the top width [31] (black line), and the CMS monotop search [19] (blue line).

constraints from single top searches can play an important role in constraining monotop scenarios. The only region in parameter space where the monotop search will always be dominant is the region where the vector is close in mass to the top, because the single top channel will be suppressed by phase space while the monotop signal is not.

\subsubsection{Dark matter constraints}

We have argued that, even for mediator masses below the top threshold, an invisible decay channel is typically needed in order for the monotop signature to be present. The simplest way out is to couple $V$ to a fermionic stable dark matter candidate $\chi$. However, in a minimal scenario where $V$ is the only mediator for the interactions of the dark matter candidate, one needs to ask whether the relic abundance of $\chi$ is enough to fulfill the bounds from observations. Below the top threshold, the main annihilation process $\chi \chi \rightarrow V \rightarrow t \bar{u}$ and $\bar{t} u$ is kinematically forbidden, so that the annihilation of dark matter particles can only proceed to a three-body or four-body final state (via a virtual top quark), or via loop-diagrams $\chi \chi \rightarrow V \rightarrow d_{i} \bar{d}_{j}$. As discussed in section 3.2.1, the loop contributions are suppressed by the mass of the light up-type quark that the mediator couples to. In section 3.1.2, we have shown that in the region where two-body final states are allowed, the relic abundance requires the couplings to be fairly large, therefore in the light mass region the $\chi \chi$ annihilation rate is, without doubts, too slow for the stable particle $\chi$ not to overpopulate the Universe.

One possible way-out is to turn on the $a_{L}$ coupling. In this case, we open back a two body decay $\chi \chi \rightarrow b \bar{d}$ and $\bar{b} d$, and similar numerical results as in section 3.1.2 at the price of a less minimal scenario. A second possibility is to have a very small $a_{R}$ so that $V$ is long lived at the price of suppressing the monotop signal beyond any hope of detectability, or complicating the dark sector so that $\chi$ is not the dark matter candidate.

Following this argument, we can state that the minimal monotop scenario is excluded by dark matter relic abundance constraints when the mediator is lighter that the top. 


\section{Conclusions}

Monotop final states comprised of a single top quark produced in association with missing energy can be a striking sign of new physics at the LHC. The main production mechanisms can be divided into two classes: resonant production, where a heavy coloured boson is first produced in the $s$-channel and further decays via its couplings to a single top quark and an invisible neutral fermion, and non-resonant production where the top quark is produced in association with an invisible boson that couples to top and up (or charm) quarks. A complete and model independent parametrisation of the two channels has been provided in ref. [8]. In the present work, we have revisited this description by embedding the effective interactions in an $\mathrm{SU}(2)_{L} \times \mathrm{U}(1)_{Y}$ invariant formalism. In doing so, we have shown that, depending on the chirality of the tops, a complete model contains necessarily extra states and couplings that may spoil the monotop signal, or add more new physics signatures that should be studied in association with the monotop one.

We have identified two minimal setups. In the first case, a scalar field is resonantly produced by the fusion of a pair of down-type quarks and couples to a right-handed top quark and a new invisible fermion, like a right-handed stop in $R$-parity violating supersymmetry. In the second case, a vector state couples to right-handed top and up quarks and decays dominantly into new invisible fields, like in models of dark matter where the dark sector couples to the Standard Model via a flavour-sensitive mediator. We have further investigated the phenomenology of the second class of models that can be split into two subclasses, depending on the mass of the mediator.

For mediators lighter than the top quark, their visible decay modes are either loopsuppressed or phase-space-suppressed, or both. Nevertheless, one always needs to add (and tune the couplings of) an invisible field to prevent the mediator from decaying inside a typical hadron collider detector as this would otherwise spoil the monotop signature originally motivating the model. An important feature of these scenarios is that they allow for the top quark to decay into the mediator and an extra jet. This feature can enhance the monotop production rate, as the monotop system can be produced in association with an extra jet from $t \bar{t}$ events when one of the top quarks decays in the exotic channel. Such events could also be searched for in standard typical single-top searches, as they are expected to populate signal regions of associated analyses. We have indeed observed that a CMS analysis of single top events could imply significant constraints on the mediator couplings, competitive and sometimes stronger than those obtained from monotop searches.

Scenarios with a mediator mass above the top threshold have a very different phenomenology as the mediator decays significantly into top quarks and jets. One needs a large coupling to the invisible sector in order to preserve the monotop signature. Describing the dark sector with a new fermion $\chi$, we have found that the latter could be a viable dark matter candidate if heavier than half the top quark mass, with a correct relic abundance driven by its annihilation via an $s$-channel mediator into a top and an up quark. We have used relic abundance constraints to derive lower bounds on the product of the couplings of the mediator to quarks and to the dark matter candidate. We have then further restricted the monotop parameter space by combining cosmological and col- 
lider results and enforcing the mediator to decay mostly invisibly. We have found that the issue of the perturbativity of the model could be raised for dark matter masses close to the top mass and that the parameter space turns out to be largely constrained when the $\chi$ fermion is demanded to reproduce the observed relic density. However, a large portion of the parameter space is still left unconstrained by current data and future experimental results are in order, in particular analyzing a same-sign top quark pair final state arising from the visible decays of the mediator.

\section{Acknowledgments}

We thank T. Theveneaux-Pelzer, J. Donini and F. Maltoni for useful discussions and comments. We acknowledge support by the Theory-LHC France initiative of CNRS/IN2P3. G.C. and A.D. acknowledge partial support from the Labex-LIO (Lyon Institute of Origins) under grant ANR-10-LABX-66 and FRAMA (FR3127, Fédération de Recherche "André Marie Ampère"). A.D. is partially supported by Institut Universitaire de France.

Open Access. This article is distributed under the terms of the Creative Commons Attribution License (CC-BY 4.0), which permits any use, distribution and reproduction in any medium, provided the original author(s) and source are credited.

\section{References}

[1] https://twiki.cern.ch/twiki/bin/view/AtlasPublic/SupersymmetryPublicResults.

[2] https://twiki.cern.ch/twiki/bin/view/CMSPublic/PhysicsResultsSUS.

[3] E.L. Berger, B.W. Harris and Z. Sullivan, Single top squark production via R-parity violating supersymmetric couplings in hadron collisions, Phys. Rev. Lett. 83 (1999) 4472 [hep-ph/9903549] [INSPIRE].

[4] F. del Aguila, J.A. Aguilar-Saavedra and L. Ametller, $Z t$ and $\gamma t$ production via top flavor changing neutral couplings at the Fermilab Tevatron, Phys. Lett. B 462 (1999) 310 [hep-ph/9906462] [INSPIRE].

[5] E.L. Berger, B.W. Harris and Z. Sullivan, Direct probes of R-parity violating supersymmetric couplings via single top squark production, Phys. Rev. D 63 (2001) 115001 [hep-ph/0012184] [INSPIRE].

[6] N. Desai and B. Mukhopadhyaya, R-parity violating resonant stop production at the Large Hadron Collider, JHEP 10 (2010) 060 [arXiv: 1002.2339] [INSPIRE].

[7] H. Davoudiasl, D.E. Morrissey, K. Sigurdson and S. Tulin, Baryon destruction by asymmetric dark matter, Phys. Rev. D 84 (2011) 096008 [arXiv:1106.4320] [INSPIRE].

[8] J. Andrea, B. Fuks and F. Maltoni, Monotops at the LHC, Phys. Rev. D 84 (2011) 074025 [arXiv: 1106.6199] [INSPIRE].

[9] J.F. Kamenik and J. Zupan, Discovering dark matter through flavor violation at the LHC, Phys. Rev. D 84 (2011) 111502 [arXiv:1107.0623] [InSPIRE].

[10] Z. Dong, G. Durieux, J.-M. Gerard, T. Han and F. Maltoni, Baryon number violation at the LHC: the top option, Phys. Rev. D 85 (2012) 016006 [arXiv:1107.3805] [inSPIRE]. 
[11] J. Wang, C.S. Li, D.Y. Shao and H. Zhang, Search for the signal of monotop production at the early LHC, Phys. Rev. D 86 (2012) 034008 [arXiv:1109.5963] [InSPIRE].

[12] B. Fuks, Beyond the minimal supersymmetric standard model: from theory to phenomenology, Int. J. Mod. Phys. A 27 (2012) 1230007 [arXiv:1202.4769] [InSPIRE].

[13] A. Kumar, J.N. Ng, A. Spray and P.T. Winslow, Tracking down the top quark forward-backward asymmetry with monotops, Phys. Rev. D 88 (2013) 075012 [arXiv: 1308.3712] [INSPIRE].

[14] E. Alvarez, E.C. Leskow, J. Drobnak and J.F. Kamenik, Leptonic monotops at LHC, Phys. Rev. D 89 (2014) 014016 [arXiv:1310.7600] [INSPIRE].

[15] J.-L. Agram, J. Andrea, M. Buttignol, E. Conte and B. Fuks, Monotop phenomenology at the Large Hadron Collider, Phys. Rev. D 89 (2014) 014028 [arXiv: 1311.6478] [InSPIRE].

[16] B. Fuks, J. Proudom, J. Rojo and I. Schienbein, Characterizing new physics with polarized beams at high-energy hadron colliders, JHEP 05 (2014) 045 [arXiv: 1403.2383] [INSPIRE].

[17] J.N. Ng and A. de la Puente, Probing radiative neutrino mass generation through monotop production, Phys. Rev. D 90 (2014) 095018 [arXiv: 1404.1415] [INSPIRE].

[18] CDF collaboration, T. Aaltonen et al., Search for a dark matter candidate produced in association with a single top quark in pp collisions at $\sqrt{s}=1.96$ TeV, Phys. Rev. Lett. 108 (2012) 201802 [arXiv:1202 .5653] [INSPIRE].

[19] CMS collaboration, Search for new physics with monotop final states in pp collisions at $\sqrt{s}=8 \mathrm{TeV}$, CMS-PAS-B2G-12-022 (2012).

[20] CMS collaboration, Search for monotop signatures in proton-proton collisions at $\sqrt{s}=8$ TeV, arXiv:1410.1149 [INSPIRE].

[21] ATLAS collaboration, Search for invisible particles produced in association with single-top-quarks in proton-proton collisions at $\sqrt{s}=8 \mathrm{TeV}$ with the ATLAS detector, arXiv:1410.5404 [INSPIRE].

[22] H. Davoudiasl, D.E. Morrissey, K. Sigurdson and S. Tulin, Hylogenesis: a unified origin for baryonic visible matter and antibaryonic dark matter, Phys. Rev. Lett. 105 (2010) 211304 [arXiv: 1008.2399] [INSPIRE].

[23] I. Jack and H. Osborn, General two loop $\beta$-functions for gauge theories with arbitrary scalar fields, J. Phys. A 16 (1983) 1101 [INSPIRE].

[24] L.-F. Li, Oblique electroweak corrections from heavy scalar fields, Z. Phys. C 58 (1993) 519 [INSPIRE].

[25] G. Bhattacharyya, A. Kundu, T. De and B. Dutta-Roy, Effects of isodoublet color-octet scalar bosons on oblique electroweak parameters, J. Phys. G 21 (1995) 153 [InSPIRE].

[26] ATLAS collaboration, Search for long-lived, multi-charged particles in pp collisions at $\sqrt{s}=7$ TeV using the ATLAS detector, Phys. Lett. B 722 (2013) 305 [arXiv:1301.5272] [INSPIRE].

[27] CMS collaboration, Searches for long-lived charged particles in pp collisions at $\sqrt{s}=7$ and 8 TeV, JHEP 07 (2013) 122 [arXiv: 1305.0491] [INSPIRE].

[28] J. Alwall et al., Computing decay rates for new physics theories with FeynRules and MadGraph5/aMC@NLO, arXiv:1402.1178 [INSPIRE]. 
[29] A. Belyaev, N.D. Christensen and A. Pukhov, CalcHEP 3.4 for collider physics within and beyond the standard model, Comput. Phys. Commun. 184 (2013) 1729 [arXiv:1207.6082] [INSPIRE].

[30] E.W. Kolb and M.S. Turner, The early universe, Frontiers in Physics volume 69, Addison-Wesley, U.S.A. (1990).

[31] CDF collaboration, T.A. Aaltonen et al., Direct measurement of the total decay width of the top quark, Phys. Rev. Lett. 111 (2013) 202001 [arXiv: 1308.4050] [INSPIRE].

[32] Particle Data Group collaboration, J. Beringer et al., Review of particle physics, Phys. Rev. D 86 (2012) 010001 [INSPIRE].

[33] CMS collaboration, Measurement of the t-channel single-top-quark production cross section and of the $\left|V_{t b}\right|$ CKM matrix element in pp collisions at $\sqrt{s}=8$ TeV, JHEP 06 (2014) 090 [arXiv: 1403.7366] [INSPIRE].

[34] ATLAS collaboration, Measurement of the t-channel single top-quark production cross section in pp collisions at $\sqrt{s}=7$ TeV with the ATLAS detector, Phys. Lett. B 717 (2012) 330 [arXiv:1205.3130] [INSPIRE].

[35] S. Kraml et al., Searches for new physics: Les Houches recommendations for the presentation of LHC results, Eur. Phys. J. C 72 (2012) 1976 [arXiv:1203.2489] [InSPIRE].

[36] N.D. Christensen and C. Duhr, FeynRules - Feynman rules made easy, Comput. Phys. Commun. 180 (2009) 1614 [arXiv:0806.4194] [INSPIRE].

[37] A. Alloul, N.D. Christensen, C. Degrande, C. Duhr and B. Fuks, FeynRules 2.0 - A complete toolbox for tree-level phenomenology, Comput. Phys. Commun. 185 (2014) 2250 [arXiv: 1310.1921] [INSPIRE].

[38] C. Degrande et al., UFO - The Universal FeynRules Output, Comput. Phys. Commun. 183 (2012) 1201 [arXiv:1108.2040] [INSPIRE].

[39] J. Alwall et al., The automated computation of tree-level and next-to-leading order differential cross sections and their matching to parton shower simulations, JHEP 07 (2014) 079 [arXiv: 1405.0301] [INSPIRE].

[40] T. Sjöstrand, S. Mrenna and P.Z. Skands, PYTHIA 6.4 physics and manual, JHEP 05 (2006) 026 [hep-ph/0603175] [INSPIRE].

[41] DELPHES 3 collaboration, J. de Favereau et al., DELPHES 3, a modular framework for fast simulation of a generic collider experiment, JHEP 02 (2014) 057 [arXiv:1307.6346] [INSPIRE].

[42] B. Dumont, B. Fuks, S. Kraml, S. Bein, G. Chalons et al., Towards a public analysis database for LHC new physics searches using MadAnalysis 5, arXiv:1407.3278 [INSPIRE].

[43] E. Conte, B. Fuks and G. Serret, MadAnalysis 5, a user-friendly framework for collider phenomenology, Comput. Phys. Commun. 184 (2013) 222 [arXiv:1206.1599] [INSPIRE].

[44] E. Conte, B. Dumont, B. Fuks and C. Wymant, Designing and recasting LHC analyses with MadAnalysis 5, Eur. Phys. J. C 74 (2014) 3103 [arXiv:1405.3982] [INSPIRE]. 